Bundesgesundheitsbl 2015 · 58:1192

DOI 10.1007/s00103-015-2228-0

Online publiziert: 8. August 2015

๑) Springer-Verlag Berlin Heidelberg 2015

CrossMark

Bekanntmachung des Umweltbundesamtes

\title{
Stellungnahme der Innenraumlufthygiene-Kommission (IRK) zu Luftreinigern
}

nach, insbesondere wenn die Adsorbentien erschöpft sind und nicht rechtzeitig gewechselt werden. Neuere Verfahren arbeiten auch mit „Luftsauerstoffaktivierung" oder „Ionisierung" der Luft. Die Hersteller versprechen dem Nutzer eine Entfernung von Luftverunreinigungen verschiedener Art, so dass ein der Gesundheit zuträgliches Raumklima entstehen soll. Einige der angebotenen Geräte verwenden nach Herstellerangaben beispielsweise einen Plasmagenerator zur Erzeugung von Ionen oder es wird explizit auf einen Ozonerzeuger hingewiesen. In Bezug auf die Wirkung sind die Herstelleraussagen unspezifisch. So sollen durch das Plasma geladene Ionen und Radikale entstehen, die zu einer Zersetzung flüchtiger organischer Verbindungen führen. Desgleichen können diese Zersetzungsprozesse auch durch Ozon oder photokatalytische Prozesse initiiert werden. Auch geruchsaktive Stoffe sollen dabei weitgehend entfernt werden. Bei allen Ansätzen ist zu beachten, dass die Reaktionsprodukte häufig unbekannt sind bzw. eine vollständige Mineralisierung (Umsetzung zu anorganischen Produkten wie $\mathrm{CO}_{2}$ und Wasser) nicht gewährleistet ist [1]. Einige Technologien verwenden chemische Wirkstoffe zur Reduzierung von Mikroorganismen. Hierbei besteht die Gefahr, dass diese Wirkstoffe in die Raumluft eingetragen werden.

Aussagen zur Wirksamkeit der Verfahren beziehen sich bisher weitestgehend auf Herstellerangaben. In der Literatur finden sich zwar zahlreiche Untersuchungen zu tragbaren Luftreinigungsgeräten auf Basis verschiedener Reinigungsverfahren [2], die Wirksamkeit dieser Verfahren wurde jedoch häufig an ausgewählten organischen Substanzen unter Laborbedingungen nachgewiesen. Unter
Realbedingungen konnten in diversen Experimenten mit Geräten, die mit Ozon oder Photokatalyse arbeiten, sogar Sekundärverbindungen in der Raumluft nachgewiesen werden, die durch Abbaureaktion oder durch Matrixabbau verursacht wurden. Weitere Studien zeigen, dass die Effekte durch die Reinigungsgeräte nicht immer vorhersagbar sind und die Luftqualität sogar verschlechtert wird oder bestenfalls gleich bleibt [3]. Standardisierte Testverfahren sind zwar in der Entwicklung, stehen bislang nur in sehr unzureichendem Maße zur Verfügung [4].

Die Innenraumlufthygienekommission stellt daher auf Basis der verfügbaren Literatur fest, dass der Einsatz von Luftreinigern derzeit bei einzelnen Verfahren zwar zu zeitlich begrenzten Reinigungsleistungen für einzelne Stoffe führen kann; manche Luftreiniger setzen jedoch nicht vorhersagbare Sekundärverbindungen in die Raumluft frei, die derzeit nicht abschließend gesundheitlich bewertet werden können. Die Kommission rät vom Einsatz solcher Geräte ab, solange es keine anerkannten standardisierten Prüfverfahren zu deren Zulassung gibt und eine Gesundheitsgefährdung für die Raumnutzer nicht in allen Fällen ausgeschlossen werden kann.

\section{Literatur}

1. Gunschera J et al (2015) Portable photocatalytic air cleaners: efficiencies and by-product generation. (Submitted for publication)

2. Luengas A et al (2015) A review of indoor air treatment technologies. Rev Environ Sci Biotechnol. doi:10.1007/s11157-015-9363-9 (available online)

3. Zeidler 0 et al (2009) Effektivität von Geräten zur Luftreinigung. Gesundheits-Ingenieur 130:3-7

4. Costarramone $\mathrm{N}$ et al (2015) Efficiency and harmfulness of air-purifying photocatalytic commercial devices: from standardized chamber tests to nanoparticles release. Catalysis Today. doi:10.1016/j.cattod.2015.01.008 (available online) 